\title{
QoS Support for Real-Time Applications Using the Integration of RSVP/Intserv and Diffserv: A Testbed Experiment
}

\author{
Seong-Ho Jeong ${ }^{1}$, Myung Choi ${ }^{1}$, Randal Abler ${ }^{1}$, Henry Owen ${ }^{1}$, John Copeland ${ }^{1}$, \\ and Joachim Sokol ${ }^{2}$ \\ ${ }^{1}$ School of Electrical and Computer Engineering, Georgia Institute of Technology, \\ Atlanta, Georgia 30332-0250, USA \\ ${ }^{2}$ Siemens AG, Corporate Technology, \\ D-81730 Munich, Germany
}

\begin{abstract}
The Integrated Services (Intserv) architecture with a resource reservation protocol such as RSVP is able to provide end-to-end quality of service (QoS) to real-time applications. However, it seems that this end-to-end model alone will not scale well for large network topologies. To overcome this scalability problem, the Differentiated Services (Diffserv) architecture has been created, which is based on the control of aggregated traffic. Diffserv, however, does not guarantee end-to-end QoS for real-time applications. Recently, lots of attention is being paid to the integration of RSVP/Intserv and Diffserv because it can provide both end-to-end QoS and scalability. This paper presents a testbed realization of the integration of RSVP/Intserv and Diffserv to provide resource reservation and end-to-end QoS to real-time applications such as voice over IP. We also present some experimental results.
\end{abstract}

\section{Introduction}

Recent research on QoS support in the Internet has led to two distinct approaches: the Integrated Services architecture (Intserv)[2] and its accompanying signaling protocol, RSVP[3], and the Differentiated Services architecture (Diffserv)[1]. The goal of Intserv is to allow end-to-end QoS to be provided to applications. The current set of services of the Intserv architecture consists of guaranteed and controlled load services. The Intserv architecture needs some explicit setup mechanism to convey information to routers so that they can provide the requested services to flows that require them. RSVP is the most common example of such a setup mechanism. RSVP is a signaling protocol used to request resources from the network. The network responds by explicitly admitting or rejecting RSVP requests [3, 4].

The current prevailing model of RSVP usage is based on a combined RSVP/Intserv architecture where RSVP signals per-flow resource requirements to network elements by using Intserv parameters as defined in the appropriate Intserv service specification 
[2]. These network elements apply Intserv admission control to signaled requests. In addition, traffic control mechanisms in each network element are configured to ensure that each admitted flow receives the service requested in strict isolation from other traffic. However, the use of per-flow state and per-flow processing in the RSVP/Intserv approach raises scalability concerns for large networks.

On the other hand, Diffserv has been motivated by the market need for immediate deployment of a QoS solution for the Internet as well as enterprise networks. In contrast to the per-flow orientation of RSVP, Diffserv networks classify packets into one of a small number of aggregated classes, based on the Diffserv codepoint (DSCP) in the packet's IP header [1]. At each Diffserv router, packets are subjected to a per-hop behavior (PHB), which is invoked by the DSCP. A PHB is defined to provide a specific forwarding behavior at each router within the Diffserv domain. Diffserv eliminates the need for per-flow state and per-flow processing and therefore scales well to large networks. However, Diffserv does not guarantee end-to-end QoS for QoSsensitive applications.

RSVP/Intserv and Diffserv can be considered as complementary technologies in the pursuit of end-to-end QoS and scalability [4]. RSVP/Intserv enables hosts to request per-flow, required resources, along end-to-end data paths and to obtain feedback regarding admissibility of these requests. Diffserv enables scalability across large networks. Therefore, the integration of Intserv/RSVP and Diffserv can provide a combination of scalability and end-to-end QoS.

This paper presents a testbed realization of the integration of RSVP/Intserv and Diffserv to provide end-to-end QoS to real-time applications such as voice over IP. We also present some experimental results. The rest of the paper is organized as follows. In section 2, we describe service definitions and service mapping options between Intserv services and Diffserv services. Section 3 presents our physical testbed structure for the integration of RSVP/Intserv and Diffserv. Section 4 shows experimental results based on different network scenarios. Finally, section 5 presents a summary of the paper and proposed future work.

\section{Service Definitions and Mapping}

The Intserv architecture specifies two service classes: guaranteed service (GS) and controlled-load service (CLS). The GS provides an assured level of bandwidth, a firm end-to-end delay bound and no queueing loss for conforming packets of a data flow. It is intended for applications with stringent real-time delivery requirements.

Unlike guaranteed service, CLS provides no firm quantitative guarantees. If the flow is accepted for controlled-load service, the router makes a commitment to offer the flow a service equivalent to that seen by a best-effort flow on a lightly loaded network. The important difference is that the controlled-load flow does not noticeably deteriorate as the network load increases. By contrast, a best-effort flow will experience progressively worse service as the network load increases. Controlled-load service is intended for those classes of applications that requires a certain level of bandwidth and can tolerate a certain amount of loss and delay. 
On the other hand, network elements within the Diffserv region select a PHB as the specific forwarding treatment to provide a specific service to incoming packets. In this paper, we consider three types of forwarding behavior: expedited forwarding (EF), assured forwarding (AF), and default forwarding for best-effort (BE) service.

The goal of the EF PHB is to provide a low loss, low latency, low jitter, assured bandwidth, and end-to-end service through the Diffserv region. Such a service appears to the endpoints like a point-to-point connection or a virtual leased line [10].

In order to create such a service, it is necessary to bound rates such that, at every transit node, the EF aggregate's maximum arrival rate is less than that EF aggregate's minimum departure rate. To do this, it is necessary to configure nodes so that the aggregate has a well-defined minimum departure rate regardless of the intensity of other traffic at the node. The EF PHB provides such a forwarding treatment for a particular Diffserv aggregate. To minimize the impact that EF traffic could have on other traffic, traffic that exceeds a certain limit must be discarded. This maximum EF rate, and burst size if appropriate, must be settable by either static or dynamic means. Several types of queue scheduling mechanisms may be employed to provide the EF PHB. In our testbed, we use class-based queueing (CBQ) [13].

The AF PHB group is a means for the Diffserv region service provider to offer different levels of forwarding assurances for IP packets received from the customer in an Intserv region [11]. Four AF classes are defined in [11], where each AF class in each Diffserv node is allocated a certain amount of forwarding resources such as buffer space and bandwidth. The details of this allocation are subject to a provider/provider or customer/provider SLS and may vary in different domains. IP packets that wish to use the services provided by the AF PHB group are assigned by the customer or the Diffserv region provider into one or more of these AF classes according to the services that the customer has subscribed to. To implement the AF PHB group, we use CBQ.

Service requests in the Intserv region specify an Intserv service type and a set of quantitative parameters known as a flowspec. For seamless operations, requests in the Intserv region need to be mapped onto the underlying capabilities of the Diffserv region. To do this, an appropriate PHB, or set of PHBs, for the requested service must be selected, and appropriate policing at the edges of the Diffserv region must be performed. Further, admission control on the Intserv requests that takes into account the resource availability in the Diffserv region should be performed.

After selecting a specific service mapping, the actual mapping of traffic parameters from the Intserv traffic specification to a specification suitable for the Diffserv domain needs to be defined. The specification in the Diffserv domain depends on the Diffserv service type, service level specification (SLS), and admission control procedures. Policy information and the amount of requested/available resources also need to be considered.

The GS requires that the Diffserv region can provide bounded delay and no queuing loss. The ingress edge router needs to know the maximum delay, and the advertised delay parameters in the adspec object of the RSVP PATH messages needs to be updated. The EF PHB is a natural choice to support the GS. In this case, however, an end-to-end characterization of the EF PHB in a Diffserv network is necessary to support end-to-end QoS. Further investigation of this mapping is under study. 
The CLS could be supported by AF PHB or EF PHB since there are no stringent QoS guarantees to be provided by the network. For AF PHB, the service provisioning or admission control procedures should make sure that the required throughput can be provided to target applications.

Based on the assumption that initial deployment of Diffserv is probably based on static provisioning, we also focus on the static case in the first stage for the mapping of GS-to-EF and CLS-to-AF. Despite the fact that other service mappings are possible, we used this straightforward mapping.

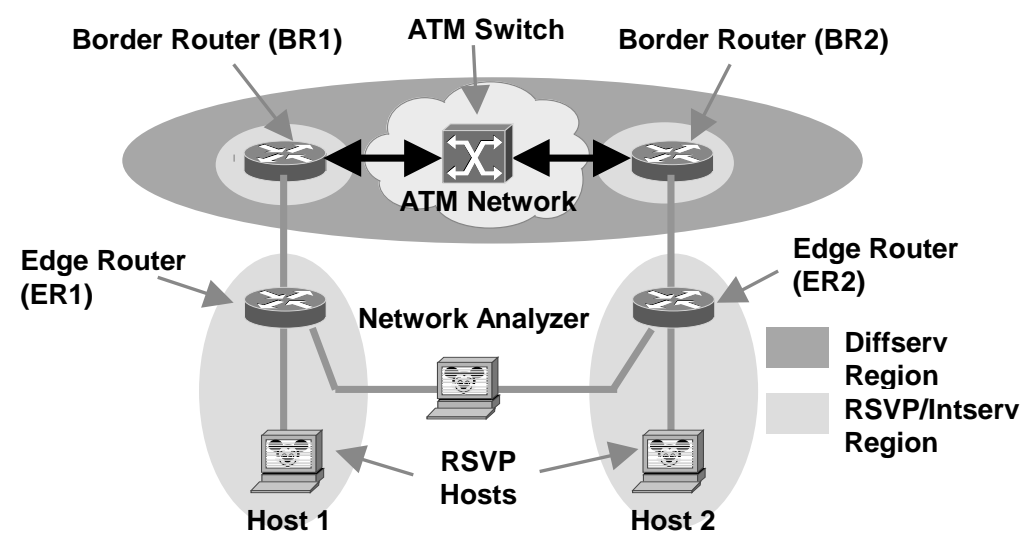

Fig. 1. Physical testbed for the integration of RSVP/Intserv and Diffserv

\section{Physical Testbed for RSVP/Intserv and Diffserv Integration}

Our physical testbed in Figure 1 is based on [4], where end-to-end QoS is provided by coupling two Intserv regions with a Diffserv region. Intserv regions are located at the periphery of the network, and the Diffserv region is in the core of the network. The edge routers at the network boundary interface to the Intserv and Diffserv regions. We use ATM as the backbone technology. Although this is not strictly necessary in order to examine the concepts presented in this paper, we believe that the use of ATM backbone will be common due to deployed infrastructure.

The Diffserv region of the network is treated as virtual links connecting Intserv capable routers. Within the Diffserv region of the network routers implement specific PHBs for aggregate traffic control. The total amount of traffic that is admitted into the Diffserv region that will receive a certain PHB is limited by policing at the edge. The Diffserv region of the network is able to support the Intserv style services requested from the periphery.

To focus on the mapping aspect and the behavior of the aggregates within the Diffserv domain, we assume that the Diffserv domain is not RSVP aware. In addition, we 
consider a single QoS sender (Host1 or Host2) in one of the Intserv regions and a single QoS receiver (Host1 or Host2) in the other.

The network elements such as routers and hosts in the testbed are based on the Linux operating system. Recent Linux kernel versions offer a wide variety of traffic control functions to support QoS [5, 6]. We utilize those traffic control functions to realize an integration of RSVP/Intserv and Diffserv. The following sections describe the testbed structure in further detail.

\subsection{Intserv and Diffserv Regions}

Each Intserv region consists of an Intserv capable host (Host1 or Host2) and an edge router (ER1 or ER2). A key requirement in the Intserv regions is that the host and edge router need to be able to process RSVP messages.

The Diffserv region supports aggregate traffic control and is assumed not to be able to support per-flow classification. As we mentioned above, devices (mainly routers) in the Diffserv region are not RSVP aware, and therefore they will pass RSVP messages transparently. The Diffserv region provides three levels of PHBs based on the DSCP in packet headers: $\mathrm{EF}, \mathrm{AF}$, and $\mathrm{BE}$.

The Diffserv region is able to provide support for the standard Intserv QoS services between its edge routers. It is also possible to invoke these services by use of standard PHBs within the Diffserv region. The Diffserv region provides admission control information to the Intserv regions through static service level specifications enforced at the edges of the Diffserv region. The Diffserv region is able to pass RSVP messages, in such a manner that they can be recovered at the egress of the Diffserv region.

\subsection{Hosts Within the Intserv Regions}

Both sending and receiving hosts (Host 1 and Host 2 in Figure 1) use RSVP to communicate the QoS requirements of QoS-aware applications running on the host. A daemon process called rsvpd is handling all RSVP signaling on behalf of these applications. In our testbed, we are using an implementation of ISI and Alexey Kuznetsov's code [7]. Figure 2 illustrates interactions between the application and rsvpd. The figure also shows the interaction between the host, edge router, and border router.

Despite the fact that the necessary marking of the IP packets with the appropriate DSCP could occur on every node (including the host) before the packet enters the Diffserv domain, we decided to use the edge router for this task, because only traffic leaving the Intserv domain must be marked. On the other hand, host marking seems to be the best way to achieve an appropriate marking from the applications point of view, but this solution suffers from the missing knowledge which service classes are provided by the network. 


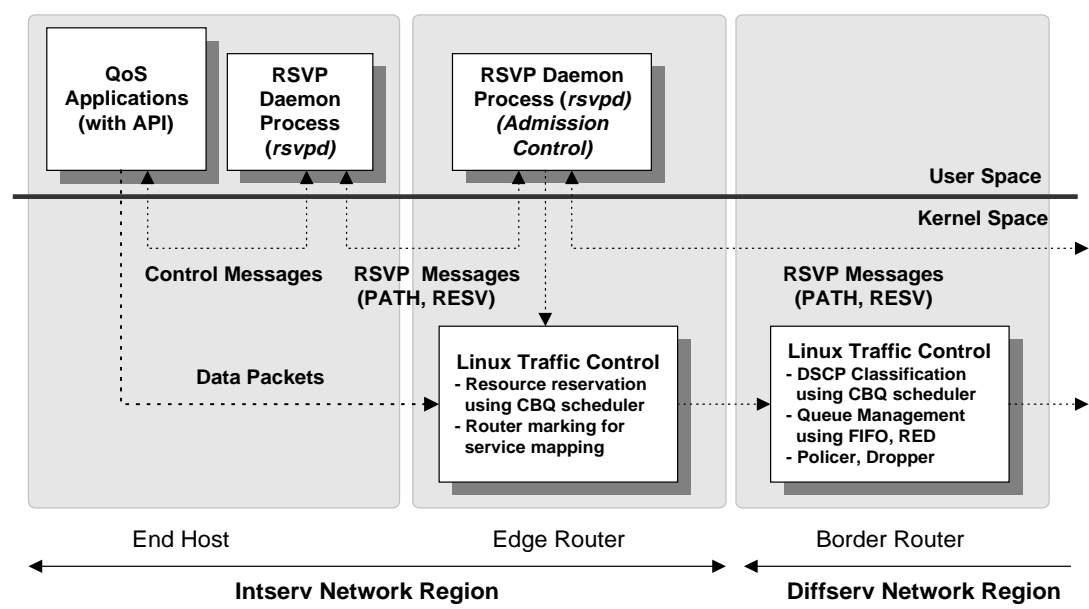

Fig. 2. Interactions between application, rsvpd process, and traffic control functions

\subsection{Edge Routers}

Edge routers (ER1 and ER2 in Figure 1) interface to both the RSVP/Intserv region and the Diffserv transit region. Edge routers are able to process PATH and RESV messages. They store RSVP state and provide per-flow classification. Thus, the edge routers are able to identify and process traffic on a per-flow granularity.

The edge router also provides the admission control function based on the transmit capacity which is statically provisioned at each Diffserv service level. To adapt a Diffserv network to new requirements, either reconfiguration of the edge routers or a more novel concept like a bandwidth broker to manage the overall network resources can be used.

In order to request resources from the network, QoS applications in the Intserv region use RSVP as an explicit signaling protocol. These requests will be accepted or rejected by our edge routers based on a local table which specifies the transmit capacity provisioned at each Diffserv service level. The network will send a rejection message in response to requests for resources that would exceed the available capacity. Consequently, the upstream host and QoS application will have the information needed to take corrective action. As a result, the integrity of those flows that were admitted can be preserved, at the expense of the flows that were not admitted. Thus, by appointing an Intserv-conversant admission control agent in the edge router for the Diffserv region of the network it is possible to enhance the service that the network can provide to QoS applications. 


\subsection{Border Routers}

Border routers (BR1 and BR2 in Figure 1) reside in the Diffserv region. In our implementation, these routers act as pure Diffserv routers since the Diffserv region is not RSVP aware. As such, their sole responsibility is to police submitted traffic based on the service level specified in the DSCP and the agreement negotiated with the customer (aggregate traffic control). RSVP messages are ignored by these routers in the Diffserv region and then processed at edge routers ER1 and ER2 according to standard RSVP processing rules.

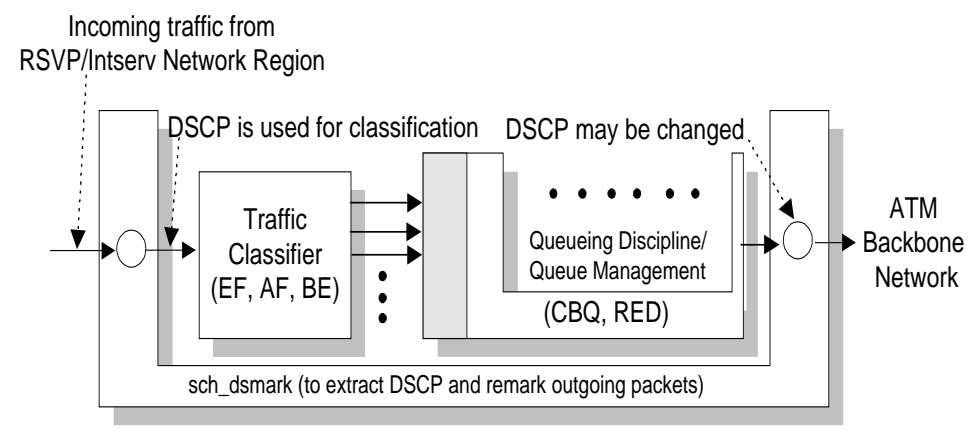

Fig. 3. The internal structure of border routers

The internal structure of border routers is shown in Figure 3, which is based on [6]. Border routers are configured to perform packet classification based on DSCP, packet scheduling, queue management, policing/dropping. The following sections describe some of the functions in further detail.

\subsubsection{Packet Classification Based on DSCP}

Within the Diffserv region, service is allocated to traffic based on the DSCP in each packet's IP header. Therefore, it is necessary to mark the DSCP correctly to obtain a particular level of service within the Diffserv region. Note that to protect Intserv traffic in Diffserv regions, DSCPs assigned to such traffic should not overlap with the DSCPs assigned to other traffic.

It is desirable to allocate service to traffic based on the application and/or user originating the traffic. Port number and source IP address in the IP headers can be used to identify packets associated with a specific application and a specific user, respectively.

\subsubsection{Packet Scheduling}

As we mentioned above, we use CBQ as our packet scheduler, which is an approach proposed in [13]. For CBQ a single set of mechanisms is proposed to implement link-sharing and real-time services. The mechanisms include a classifier to classify 
arriving packets to the appropriate class, an estimator to estimate the bandwidth recently used by a class, a selector to determine the order in which packets from the various classes will be sent, and a delayer or overlimit action to schedule traffic from classes that have exceeded their link-sharing limits and are contributing to congestion. In our implementation, $\mathrm{CBQ}$ is used to classify $\mathrm{EF}, \mathrm{AF}$, and $\mathrm{BE}$ traffic so that each user can get appropriate resources based on the user service profile.

\subsubsection{Queue Management}

An AF implementation must attempt to minimize long-term congestion with each class, while allowing short-term congestion resulting from bursts. This requires an active queue management algorithm.

We consider here only random early detection (RED) as an active queue management algorithm for routers. In contrast to traditional queue management algorithms, which drop packets only when the buffer is full, the RED algorithm drops arriving packets probabilistically [14]. The probability of drop increases as the estimated average queue size grows. Note that RED responds to a time-averaged queue length, not an instantaneous one. Thus, if the queue has been mostly empty in the "recent past", RED won't tend to drop packets unless the queue overflows. On the other hand, if the queue has recently been relatively full, indicating persistent congestion, newly arriving packets are more likely to be dropped. The RED algorithm itself consists of two main parts: estimation of the average queue size and the decision of whether or not to drop an incoming packet. In our testbed, RED is attached to the classes created for BE as well as AF.

\subsection{ATM Switch}

An ATM switch is the only component of our ATM backbone and provides highspeed connectivity within the Diffserv region. The ATM switch is also used to create congestion in our testbed by assigning a limited bandwidth to a permanent virtual circuit (PVC). To connect the border routers with the ATM switch, we use Classical IP over ATM (CLIP) which is defined in RFC1577.

\section{Experimental Results}

We consider voice-over-IP (VoIP) traffic as a real-time traffic source in our testbed. We are using a network analyzer to generate simulated VoIP traffic. Voice is divided into small time windows, called frames, and the voice samples comprising each frame are encoded for transmission. For transmission of voice flows over IP networks, one or more frames are carried in each IP packet. We call this the packetization of the voice signal. The header bytes often imply a substantial bandwidth overhead for carrying voice over IP networks. 


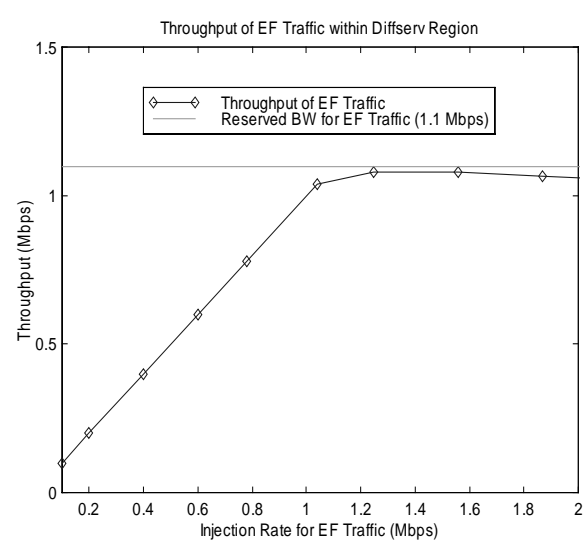

(a)

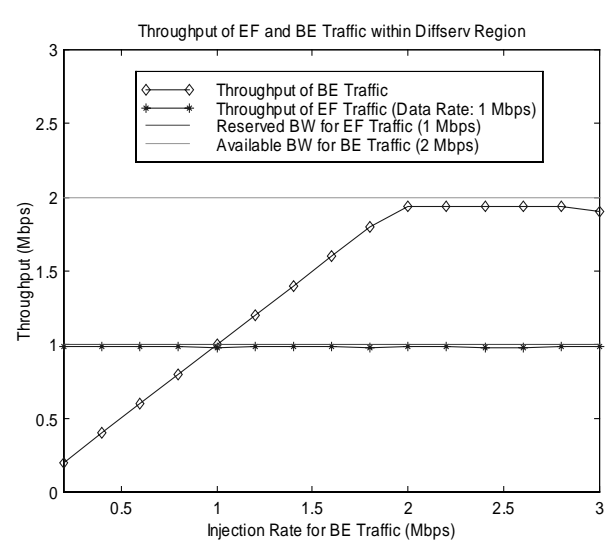

(b)

Fig. 4. (a) Throughput of EF traffic within the Diffserv region (b) Throughput of EF and BE traffic within the Diffserv region

Because packets of a VoIP flow using UDP flow can be dropped by an IP network due to congestion or arrive out of sequence at the destination, an additional protocol called real-time transport protocol (RTP) is necessary to enable receivers to reorder out-of-sequence packets and to detect packets, which are outside the time window for processing. Therefore RTP is adding a sequence number and a timestamp to every packet.

For our experimental studies, we assume that each VoIP packet encodes two $10 \mathrm{~ms}$ G.729 frames, for a total of $20 \mathrm{~ms}$ of speech. The VoIP packet size is 60 bytes, which includes 20 bytes for the two G.729 frames plus an overhead of 40 bytes ( 20 bytes for IP header, 8 bytes for UDP header, and 12 bytes for RTP header).

Figure 4-(a) shows how well our testbed can support the EF PHB within the Diffserv region. In this experiment, we use one aggregate traffic source which generates simulated 60-byte VoIP packets marked with EF DSCP (101110). The Diffserv routers (BR1 and BR2 in Figure 1) maintain a separate queue for EF class. The aggregate rate at which the traffic source produces EF-marked packets ranges from 0.1 Mbps to 2.0 Mbps. The reserved bandwidth in the Diffserv region for EF-marked packets is 1.1 Mbps. As shown in Figure 4-(a), EF-marked traffic gets the desired throughput performance until the injection rate is approximately $1.1 \mathrm{Mbps}$. Note that the traffic which exceeds the reserved bandwidth must be discarded in order to minimize the impact that EF traffic could have on other traffic.

Figure 4-(b) shows throughput performance when BE packets and EF-marked packets are transmitted simultaneously over the Diffserv region. Reserved bandwidth for EF-marked traffic is $1 \mathrm{Mbps}$, and EF-marked packets are generated at the rate of 1 Mbps. The BE packets are marked with BE DSCP (000000). The aggregate rate at which the $\mathrm{BE}$ traffic is generated ranges from $0.2 \mathrm{Mbps}$ to $3 \mathrm{Mbps}$, and available bandwidth for BE traffic is $2 \mathrm{Mbps}$. RED is used as an active queue management scheme for BE traffic. The goal of this experiment was to explore the impact of BE 
traffic on the EF-marked traffic. As shown in Figure 4-(b), EF-marked traffic gets the desired throughput behavior. Furthermore, the increase in the injection rate for $\mathrm{BE}$ traffic does not have any serious impact on the throughput performance of EF-marked traffic.

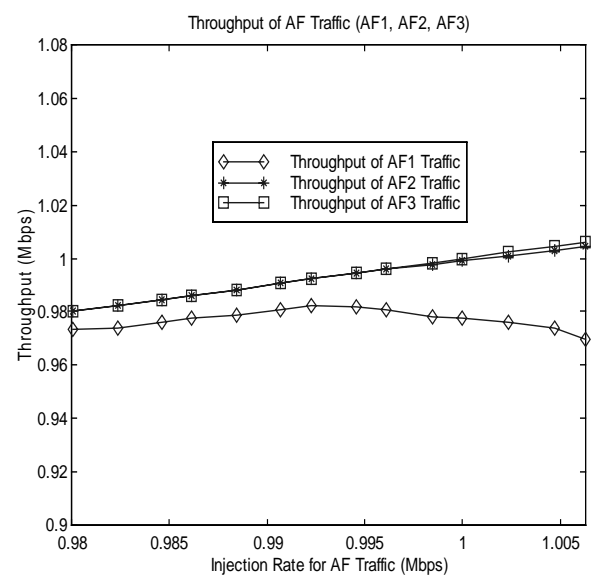

Fig. 5. Throughput of AF traffic within the Diffserv region

Figure 5 illustrates the throughput behavior of AF-marked traffic. In this experiment, we consider 3 AF classes, AF1, AF2, and AF3. Aggregate traffic for each class is generated by an independent traffic source and is marked with a corresponding AF DSCP (AF1: 001110, AF2: 010110, AF3: 011110). For simplicity, we only consider a single drop precedence level within each AF class. All traffic sources generate AFmarked packets at the same rate, and the rate ranges from $0.98 \mathrm{Mbps}$ to $1.006 \mathrm{Mbps}$. The priority levels 5, 4, and 3 are assigned to AF1 class, AF2 class, and AF3 class, respectively. That is, AF3 class is given the highest priority, and AF1 class is given the lowest priority. This means that when packets with different priority levels arrive at the Diffserv router simultaneously, the packets with an assigned lower priority level are served first. RED is used as an active queue management scheme for all AF classes. As shown in Figure 5, the AF1-marked traffic gets the lowest throughput performance as we expected.

As discussed in Section 2, GS can be mapped onto the EF PHB. Figure 6 shows throughput performance of RSVP/Intserv GS traffic when GS-to-EF service mapping is used. The aggregate rate for GS traffic is $300 \mathrm{Kbps}$ and reserved bandwidth for GS traffic is $310 \mathrm{Kbps}$. Maximum output link capacity of BR1 is artificially limited to 2.5 Mbps. Approximately 2.2 Mbps is available to BE traffic. To examine the impact that the BE traffic could have on the RSVP/Intserv traffic, injection rate for BE traffic ranges from 0.2 Mbps to $3 \mathrm{Mbps}$. As shown in Figure 6, the RSVP/Intserv traffic gets the desired throughput performance. Furthermore, an increase in the injection rate for $\mathrm{BE}$ traffic does not have any serious impact on the RSVP/Intserv traffic. 


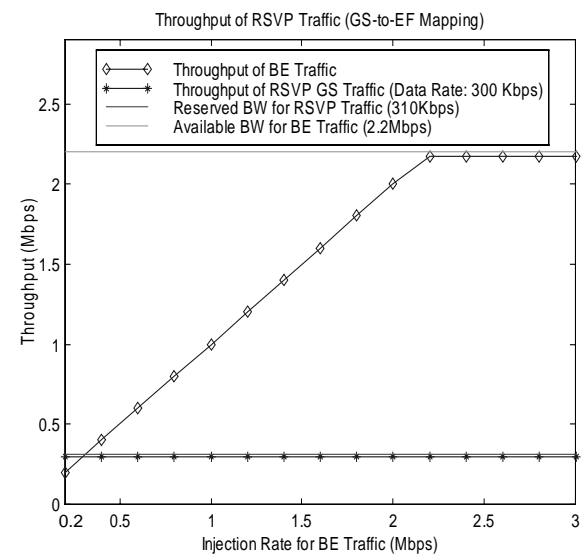

Fig. 6. Throughput of RSVP/Intserv GS traffic (GS-to-EF mapping)

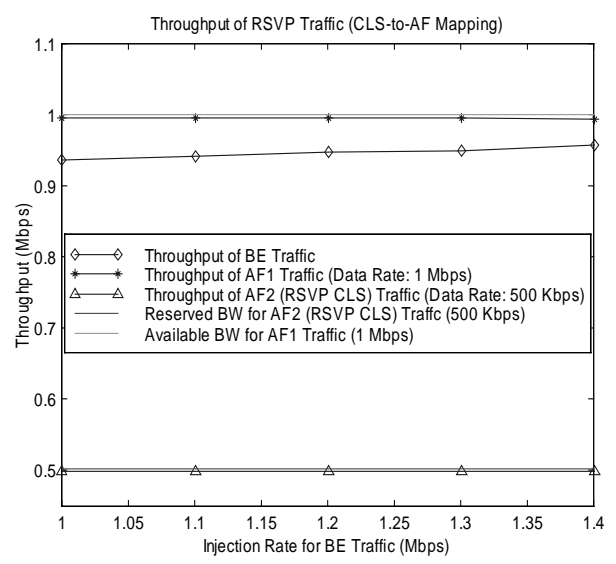

Fig. 7. Throughput of RSVP/Intserv CLS traffic (CLS-to-AF mapping)

Similarly, the AF PHB can be used to support CLS. Figure 7 shows the throughput performance of RSVP/Intserv CLS traffic when a CLS-to-AF service mapping is used. In this experiment, we create two AF classes in the Diffserv region: AF1 and AF2. We also create a $\mathrm{BE}$ class for best-effort traffic. AF2 class is given higher service priority than AF1 and BE classes. To protect CLS traffic from other traffic, CLS is mapped onto the AF2 class. The aggregate data rate for CLS traffic is $500 \mathrm{Kbps}$, and the reserved bandwidth for the CLS traffic is $500 \mathrm{Kbps}$. The aggregate data rate for AF1marked traffic is $1 \mathrm{Mbps}$, and the available bandwidth for AF1-marked traffic is 1 Mbps. The maximum output link capacity of BR1 is deliberately limited to 2.5 Mbps. To explore the impact that the BE traffic has on the CLS traffic, the injection rate for $\mathrm{BE}$ traffic ranges from 1 Mbps to 1.4 Mbps. As shown in Figure 7, CLS traffic gets the requested throughput. In addition, an increase in the injection rate for BE traffic does not have any serious impact on the CLS traffic. Compared to BE traffic, AF1marked traffic gets better performance as expected.

\section{Conclusions and Future Work}

This paper presented a testbed realization of the integration of RSVP/Intserv and Diffserv. There is still a great deal of research underway to determine how Integrated and Differentiated Services should and will interoperate. In the work presented here we used a mapping of guaranteed service into the expedited forwarding per-hop behavior. We also used a mapping of controlled-load service into the assured forwarding perhop behavior, and best-effort traffic was marked with a default differentiated service code point. 
In our Linux-based testbed implementation, we used class-based queueing to classify traffic so that each user got the appropriate resources. We also used random early detection as our active queue management algorithm for the assured forwarding and best effort classes. We used a SmartBits 2000 traffic generator to generate simulated time-sensitive voice-over-IP packets. We then made measurements of the performance of the testbed. We used Pentium II-based 300MHz machines.

Now that we have an interoperating integrated and differentiated service testbed, we plan to investigate other possible mapping options, dynamic service provisioning, enhanced admission control procedures, and detailed performance issues.

\section{Acknowledgements}

This work was partially supported by grants from Hitachi Telecom and Siemens.

\section{References}

1. Blake, S. et al: An Architecture for Differentiated Services. RFC 2475, December 1998.

2. Braden, R., Clark, D., Shenker, S.: Integrated Services in the Internet Architecture: an Overview. Internet RFC 1633, June 1994

3. Braden, R., Zhang, L., Berson, S., Herzog, S., Jamin, S.: Resource Reservation Protocol (RSVP) Version 1 Functional Specification. RFC 2205, Proposed Standard, Sep. 1997

4. Bernet, Y., Yavatkar, R., Ford, P., Baker, F., Zhang, L., Speer, M., Braden, R., Davie, B., Wroclawski, J., Felstaine, E.: A Framework for Integrated Services Operation over Diffserv Networks. Internet Draft, draft-ietf-issll-diffserv-rsvp-03.txt, Sep. 1999

5. Almesberger, W., et al: Linux Traffic Control - Implementation Overview. http://lrcwww.epfl.ch/linux-diffserv/, Nov. 1998.

6. Almesberger, W., Salim, J.H., Kuznetsov, A.: Differentiated Services on Linux. Internet Draft, draft-almesberger-wajhak-diffserv-linux-01.txt, June 1999.

7. University of Southern California (USC)/Information Sciences Institute (ISI): RSVP Software. http://www.isi.edu/div7/rsvp/release.html.

8. Guerin, R., Blake, S., Herzog, S.: Aggregating RSVP based QoS Requests. Internet Draft, draft-guerin-aggreg-rsvp-00.txt, Nov. 1997.

9. Nichols, K., et al: Definition of the Differentiated Services Field (DS Field) in the IPv4 and IPv6 Headers. RFC 2474, Dec. 1998.

10. Jacobson, V., Nichols, K., Poduri, K.: An Expedited Forwarding PHB. IETF RFC 2598, February, 1999

11. Heinanen, J., Finland, T., Baker, F., Weiss, W., Wroclawski, J.: Assured Forwarding PHB Group. IETF RFC 2597, Feb. 1999.

12. Nichols, K., Jacobson, V., Zhang, L.: A Two-bit Differentiated Services Architecture for the Internet. Internet Draft, draft-nichols-diff-svc-arch-01.txt, Apr. 1999.

13. Floyd, S., Jacobson, V.: Link-sharing and Resource Management Models for Packet Networks. IEEE/ACM Transactions on Networking, Vol. 3, No. 4, pp. 365-386, Aug. 1995.

14. Floyd, S., Jacobson, V.: Random Early Detection gateways for Congestion Avoidance. IEEE/ACM Transactions on Networking, Vol.1, No.4, pp. 397-413, Aug. 1993. 